\title{
Ultimate Limit in the Spectral Resolution of Extreme Ultraviolet Frequency Combs
}

\author{
C. Corsi, ${ }^{1}$ I. Liontos, ${ }^{1, \dagger}$ M. Bellini, ${ }^{1,2,3}$ S. Cavalieri, ${ }^{1,2}$ P. Cancio Pastor, ${ }^{1,2,3}$ M. Siciliani de Cumis, ${ }^{1,3,4}$ and R. Eramo ${ }^{1,2,3, *}$ \\ ${ }^{1}$ European Laboratory for Non-Linear Spectroscopy (LENS), Via N. Carrara 1, I-50019 Sesto Fiorentino, Firenze, Italy \\ ${ }^{2}$ Dipartimento di Fisica e Astronomia, Università di Firenze, Via G. Sansone 1, I-50019 Sesto Fiorentino, Firenze, Italy \\ ${ }^{3}$ Istituto Nazionale di Ottica (INO-CNR), Largo E. Fermi 6, I-50125 Firenze, Italy \\ ${ }^{4}$ Agenzia Spaziale Italiana (ASI), Contrada Terlecchia, I-75100 Matera, Italia
}

(Received 26 October 2016; revised manuscript received 8 February 2017; published 3 April 2017)

\begin{abstract}
We present the results of direct interferometric measurements on the pulse-to-pulse phase jitter of a metrological, fiber-based, infrared (IR) frequency comb. We show that the short-time evolution of such phase fluctuations, which cannot be actively controlled by any feedback system, imposes a stringent limit on the tooth linewidth of extreme ultraviolet (XUV) combs produced by high-order harmonic conversion, thus explaining the difference of 9 orders of magnitude between the coherence times of state-of-the-art IR and XUV frequency combs.
\end{abstract}

DOI: 10.1103/PhysRevLett.118.143201

At the turn of the 20th century, the field of optical spectroscopy was revolutionized by the invention of femtosecond optical frequency combs (OFCs) [1-5]. An OFC is based on a mode-locked ultrafast laser in which light pulses of subpicosecond duration circulate inside an optical cavity [2]. The laser thus emits a regular pulse train at a repetition rate that is determined by the time $T_{\text {rep }}$ the pulses take to make a round trip. Ideally, the phase of each pulse is shifted from that of the subsequent one by a fixed given amount $\psi_{\mathrm{ML}}$ (the mode-locking condition). These two properties result in a comb of sharp spectral lines uniformly spaced in frequency: $\nu_{n}=n \nu_{\text {rep }}+\nu_{\text {off }}$, where the repetition rate $\nu_{\text {rep }}=1 / T_{\text {rep }}$ and the offset $\nu_{\text {off }}=\psi_{\mathrm{ML}} \nu_{\text {rep }} / 2 \pi$ are radio frequencies, $\nu_{n}$ is the optical frequency of a given comb tooth and in the optical domain $n$ is an integer of the order of $10^{6}-10^{7}$. The regular comb of spectral teeth of a modelocked laser spectrum can thus be used as an absolute frequency ruler [6,7] or as a multiwavelength laser source for spectroscopic applications [8-13]. The ultimate frequency precision and spectral resolution in these OFC applications are determined by the tooth linewidth, which is $\Delta \nu \approx \nu_{\text {rep }} / N$, where $N$ is the number of comb pulses that are coherently involved in the measurement. Each OFC is thus characterized by a maximum number $N_{\text {eff }}$ of consecutive mutually phase-coherent pulses that, in the freerunning case, may be of the order of $10^{4}$, corresponding to a coherence time around $100 \mu \mathrm{s}$. Controlling the phase diffusion with a feedback loop operating on these temporal scales is not a difficult technical task and $N_{\text {eff }}$ values that are orders of magnitudes larger than the free-running ones can thus be obtained. State-of-the-art fiber-based OFCs in the optical region have indeed reached submillihertz comb tooth linewidths $[14,15]$, corresponding to $N_{\text {eff }} \sim 10^{11}$.

The situation is quite different in the extreme ultraviolet (XUV) region, where applications of frequency combs span from precision spectroscopy of hydrogen and hydrogenlike ions as fundamental tests of quantum electrodynamics, to the development of novel optical nuclear clocks $[16,17]$. The coherence of XUV combs plays a fundamental role in all these cases, and the question about its intrinsic or technical nature is still a critical and outstanding problem. XUV combs with tooth linewidths at the $\mathrm{MHz}$ level have been produced using high-order harmonic generation (HHG) from a near-infrared (NIR) OFC [12]. A linewidth $\Delta \nu \approx 1 \mathrm{MHz}$ corresponds to coherence times of $\approx 1 \mu \mathrm{s}$ and, for $\nu_{\text {rep }} \approx 100 \mathrm{MHz}$, to a rather low number of coherent XUV pulses $N_{\text {eff }} \approx 100$ [18].

In this paper we address the dramatic difference-more than 9 orders of magnitude-between the linewidths of state-of-the-art experimental realizations of OFCs in the NIR and the XUV spectral regions. We find that it is strictly related to the pulse-to-pulse phase jitter of the driving laser pulse train and to the impossibility of controlling its diffusion on sufficiently short time scales.

Figure 1 schematically illustrates this effect on a train of successive fundamental laser pulses and on the corresponding train of harmonic pulses. Taken the first pulse as a reference, the accumulation of the pulse-to-pulse phase jitter $\sigma_{1}$ over successive pulses leads to a standard deviation of $\sigma_{k}$ in the relative phase between the reference and the $k$ th pulse. $N_{\text {eff }}$ can thus be defined by $\sigma_{N_{\text {eff }}} \approx 1 \mathrm{rad}$, corresponding to the value of $k$ for which the maximum acceptable dither of the field oscillation compared to the optical period is reached. As seen above, $N_{\text {eff }}$ for freerunning mode-locked lasers is usually sufficiently large for a fast electronic feedback to avoid substantial phase diffusion. However, the same is not true for high-order harmonics generated by such pulses. Even if the HHG process does not introduce additional phase disturbances [21], the phase dither of the harmonic pulses unavoidably scales with the order $n_{h}$, as shown in the last row of Fig. 1. Therefore, a destructive phase dither may accumulate in the 


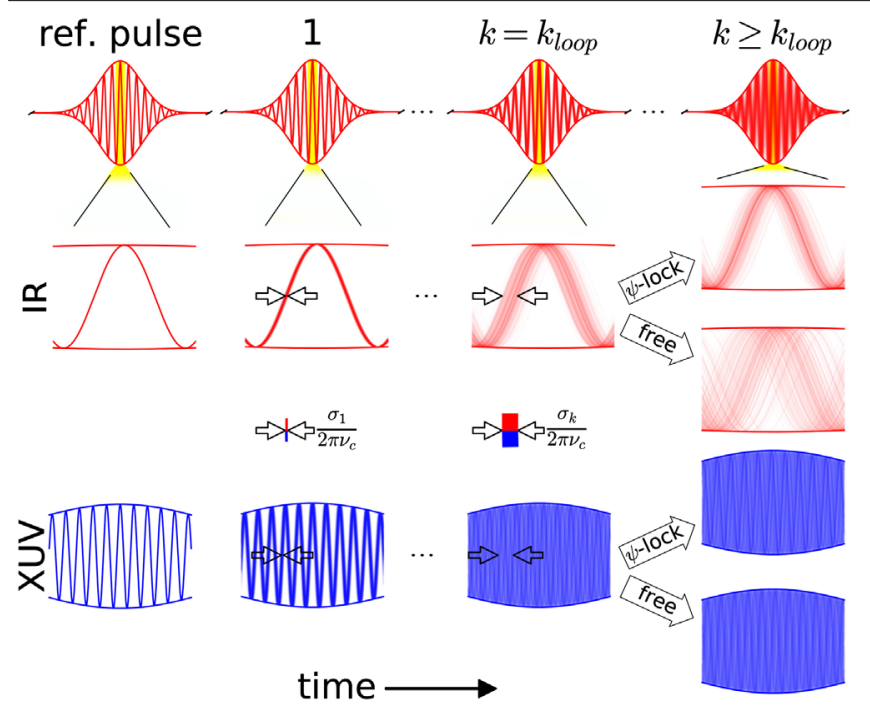

FIG. 1. Effect of the accumulated pulse-to-pulse phase jitter on successive pulses in a mode-locked IR train and on its harmonic of order $n_{h}$ (here $n_{h}=9 ; \nu_{c}$ is the IR carrier frequency). For simplicity, we show the case of zero offset frequency. In the case shown, at $k_{\text {loop }}$ (the phase-stabilization loop characteristic time in number of pulses), the harmonic field is already at its coherence limit. For $k>k_{\text {loop }}$ the effect of the stabilization loop is thus visible only on the IR pulses.

harmonic pulse train much before any feedback system has had time to counteract it. In such a case, even if the driving OFC were accurately phase stabilized to sub- $\mathrm{mHz}$ tooth linewidths, the corresponding harmonic XUV comb would essentially behave as a free-running one.

To our knowledge, the issue of phase fluctuations in an OFC train at the pulse-to-pulse level has never been experimentally addressed before [22] and, due to its crucial importance in the potentially wide range of future XUV applications, we chose to investigate it by means of an interferometric scheme. The output from a fiber-based OFC is sent through an ultrastable Michelson interferometer with an optical path difference that allows the temporal overlap and interference between consecutive pulses of the OFC train at its two (backward and forward) outputs. We use a commercial metrological OFC based on a $\mathrm{Er}^{+}$-doped fs mode-locked laser emitting around $1.55 \mu \mathrm{m}$, with a repetition rate $\nu_{\text {rep }}=250 \mathrm{MHz}$, which is expected to show a very stable phase behavior. Sequences of thousands of consecutive pulses are then detected in the time domain at the interferometer outputs in the forward and backward directions [18].

In general, the phase difference between two successive pulses can be expressed as

$$
\psi_{n+1}-\psi_{n}=\psi_{\mathrm{ML}}+\Delta \psi_{\mathrm{r}}^{(n)}
$$

where the additional pulse-to-pulse phase jitter $\Delta \psi_{\mathrm{r}}^{(n)}$ has a null average and the standard deviation $\sigma_{1}$. In principle, the analysis of the signals corresponding to the pulse trains at the two outputs of the Michelson interferometer allows us to straightforwardly recover the pulse-to-pulse intensity and phase fluctuations of the frequency comb. In fact, in the case of ideal balanced beam splitting, the $n$th and the $(n+1)$ th pulse of the OFC train completely overlap in time after having traveled, respectively, the long and short interferometer arms, to finally give output pulse energies in the forward $(f)$ and backward $(b)$ directions that can be simply expressed, in conditions of interference, as

$\mathcal{E}_{f, b}^{(n, n+1)}=\frac{1}{4}\left[\mathcal{E}_{n}+\mathcal{E}_{n+1} \pm 2 \sqrt{\mathcal{E}_{n} \mathcal{E}_{n+1}} \cos \left(\Delta \psi_{n, n+1}\right)\right]$

where $\Delta \psi_{n, n+1}=\psi_{n+1}-\psi_{n}-2 \pi \nu_{c} \Delta t, \nu_{c}$ being the comb carrier frequency and $\Delta t$ the interferometer path delay. Close to a zero crossing of the interference fringes [when the cosine term in Eq. (2) has a linear dependence on $\Delta \psi_{n, n+1}$ at the first order], a pulse-to-pulse phase jitter results in anticorrelated noise contributions in the two outputs. On the other hand, when the delay between the two arms of the interferometer is off the OFC interpulse delay $T_{\text {rep }} \approx 4$ ns [when the interference term in Eq. (2) is not present and thus $\mathcal{E}_{f}^{(n, n+1)}=\mathcal{E}_{b}^{(n, n+1)}$ ], the signals in the forward and backward outputs show correlated fluctuations, due to energy fluctuations in the input pulses. In the real experimental situation involving nonideal beamsplitting, losses, and different detection efficiencies in the two photodetectors, the signal analysis is more complex, but a full treatment with a recursive analysis of the two interferometer output trains nevertheless allows one to recover the pulse-to-pulse intensity and phase fluctuations of the input OFC pulse train [24].

Measurements were taken at three different output power levels of the $\mathrm{OFC}$, corresponding to the minimum $(55 \mathrm{~mW})$, intermediate $(200 \mathrm{~mW})$, and maximum OFC amplification settings $(380 \mathrm{~mW})$. In each condition, 3200 consecutive pulses were acquired for the two outputs of the Michelson interferometer at the maximum sampling rate of the oscilloscope [18]. Within each acquisition period, lasting $\approx 13 \mu \mathrm{s}$, the active stabilization loops of the interferometer and of the OFC have no effect. Moreover, it is still completely safe to neglect the passive instabilities (in the $\Delta t$ term of $\Delta \psi_{n, n+1}$ ) of the interferometer at these time scales.

The two-dimensional plots in the top row of Fig. 2 show the raw measured signals for the forward vs backward interferometer outputs (i.e., $\mathcal{E}_{f}^{(n, n+1)}$ vs $\mathcal{E}_{b}^{(n, n+1)}$ ), as a quick visual check of their correlations. As expected from the rough considerations outlined above, we only find small positive correlations for the noninterfering conditions, whereas statistically significant negative correlations due to pulse-to-pulse phase fluctuations are observed in all the interfering cases. A full data analysis is then performed in order to extract quantitative figures about such fluctuations. The histograms of the second row in Fig. 2 show the 


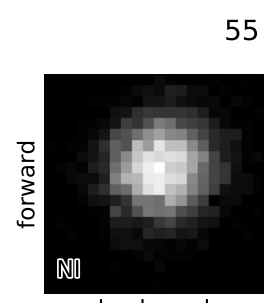

$55 \mathrm{~mW}$
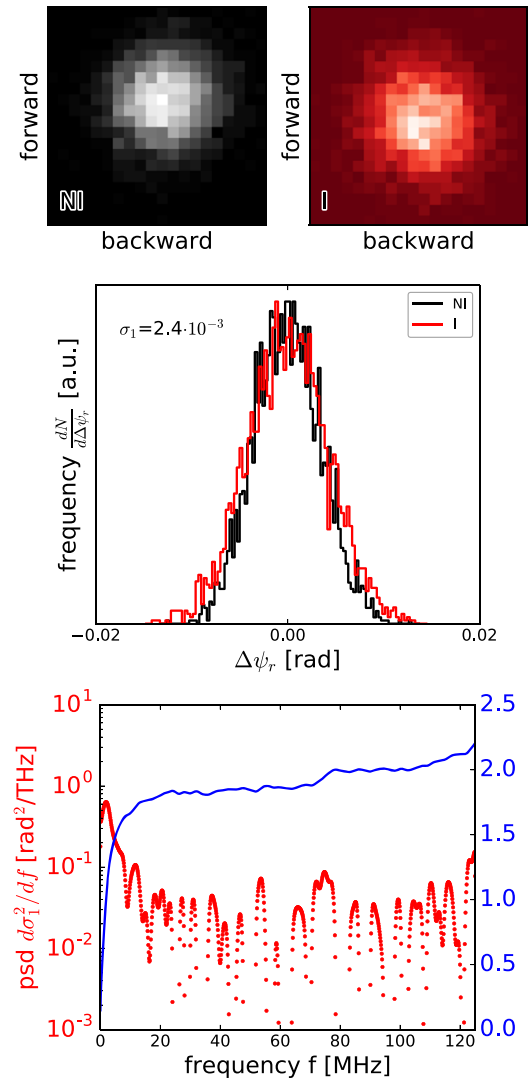

$200 \mathrm{~mW}$
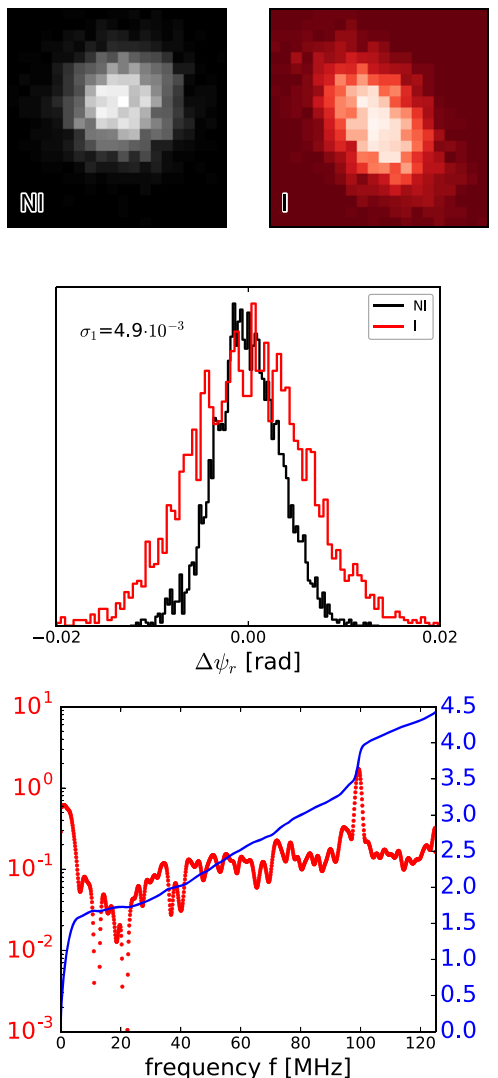

$380 \mathrm{~mW}$
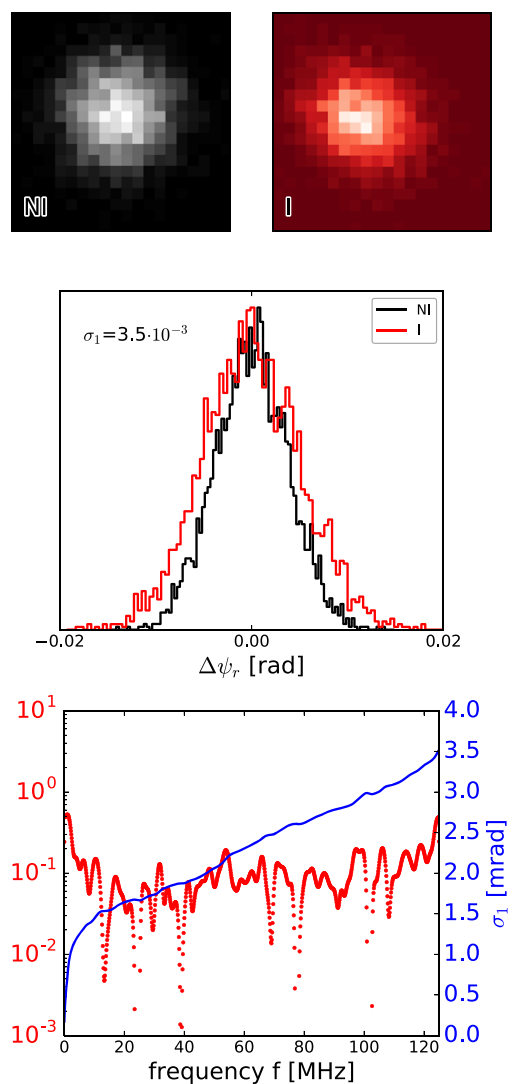

FIG. 2. 2D histograms on the top: Michelson outputs signals (experimental values of the forward vs backward pulse energies) for noninterfering (NI) and interfering conditions (I). The three couples are for representative comb regimes: $55 \mathrm{~mW}$ power (amplifier at minimum), $200 \mathrm{~mW}$ power (intermediate amplification), and $380 \mathrm{~mW}$ power (amplifier at maximum). For each couple, in the second row the recovered phase histograms of the input comb pulse train is shown. Finally, the third row shows the power spectral distribution of the phase fluctuation (left scale) and, in the right scale, the cumulative standard deviation as a function of the Fourier frequency.

distributions of the reconstructed $\mathrm{OFC}$ interpulse phase differences. Although the histograms for the noninterfering cases should ideally be constituted by $\delta$ functions, they actually present a finite and approximately constant standard deviation $\sigma_{\mathrm{NI}}$ due to residual instrumental noise. However, when successive pulses are made to interfere at the outputs of the interferometer, the corresponding distributions are clearly seen to broaden with varying standard deviations $\sigma_{\mathrm{I}}$ for the different $\mathrm{OFC}$ power levels. Because these histograms are a convolution of the OFC interpulse phase jitter distributions and of the instrumental background, which we may assume as Gaussian and uncorrelated, the standard deviation $\sigma_{1}$ of the OFC pulse-to-pulse phase differences is simply estimated as the quadratic difference between the standard deviations of the two corresponding distributions, i.e., $\sigma_{1}=\sqrt{\sigma_{\mathrm{I}}^{2}-\sigma_{\mathrm{NI}}^{2}}$. A deeper insight can be gained from the power spectral distributions (PSD) $d \sigma_{1}^{2}(f) / d f$ of the reconstructed OFC interpulse phase differences shown in the bottom row of Fig. 2 (left scale) [25]. The cumulative standard deviation $\sigma_{1}(f)=\sqrt{\int_{0}^{f} d f^{\prime} d \sigma_{1}^{2}\left(f^{\prime}\right) / d f^{\prime}}$ is also shown on the same plots (right scale); its value for a Fourier frequency corresponding to the Nyquist value $f=125 \mathrm{MHz}$, is in good agreement with the simple pulse-to-pulse standard deviation $\sigma_{1}$ found above. The OFC at the minimum amplification level presents the lowest pulse-to-pulse phase jitter of about $2 \mathrm{mrad}$; this value is entirely due to fluctuations with Fourier frequencies below the resolution bandwidth of our analysis $(2 \mathrm{MHz})$ and is common to all amplification conditions [18]. Fluctuations of about $3.5 \mathrm{mrad}$ are observed in the regime of maximum OFC amplification, where the additional $1.5 \mathrm{mrad}$ are due to a flat distribution of high-frequency noise. In the intermediate amplification case, a further $\approx 1 \mathrm{mrad}$ contribution is due to a spurious $100 \mathrm{MHz}$ oscillation. Note that this level of phase stability between consecutive pulses in an OFC is perfectly adequate for Ramsey-type spectroscopy also when performed in the XUV region in combination with HHG [26-30]. In fact, even if the measured pulse-to-pulse phase jitter of the laser is multiplied by the order $n_{h}$ of the 
harmonic, it may become a significant fraction of an XUV optical cycle, and thus destroy the visibility of Ramsey fringes, only for very high harmonic orders $\left(n_{h}>300\right)$, for such a high-quality oscillator.

Since our approach is able to completely recover the phase differences between any pair of consecutive comb pulses, it also allows us to calculate phase differences, $\psi^{(n)}\left(k T_{\text {rep }}\right)$, between two nonconsecutive ones:

$$
\psi^{(n)}\left(k T_{\text {rep }}\right)=\psi_{n+k}-\psi_{n}=k \psi_{\mathrm{ML}}+\sum_{m=0}^{k-1} \Delta \psi_{\mathrm{r}}^{(m)} .
$$

The standard deviation $\sigma_{k}$ of this quantity grows as a function of the delay $k$ due the phase diffusion term that accumulates over successive pulses. For each delay $k$, it is calculated by performing a running sum of $k$ successive terms over the recovered $\Delta \psi_{r}^{(m)}$ and a quadratic difference between the I and NI cases. The retrieved values of $\sigma_{k}$ are then plotted as a function of the delay in Fig. 3 for the three cases of Fig. 2. Two power-law reference behaviors $\sigma_{k} \propto k^{\alpha}$ are also reported in Fig. 3: $\alpha=1 / 2$, a random walk phase diffusion corresponding to a case where the $k$ terms in the sum (3) are uncorrelated, and $\alpha=1$, the linear growth indicating perfect correlation between successive phase jumps. The observed growth rate means that the fluctuations in subsequent round trips are highly correlated (i.e., $\left\langle\Delta \psi_{r}^{(n)} \Delta \psi_{r}^{(m)}\right\rangle \approx \sigma_{1}^{2}$, at least for $m$ not too far from $n$ ), and this is connected to the low-frequency peak in the PSDs of Fig. 2. If this growth were extrapolated to higher $k$, the

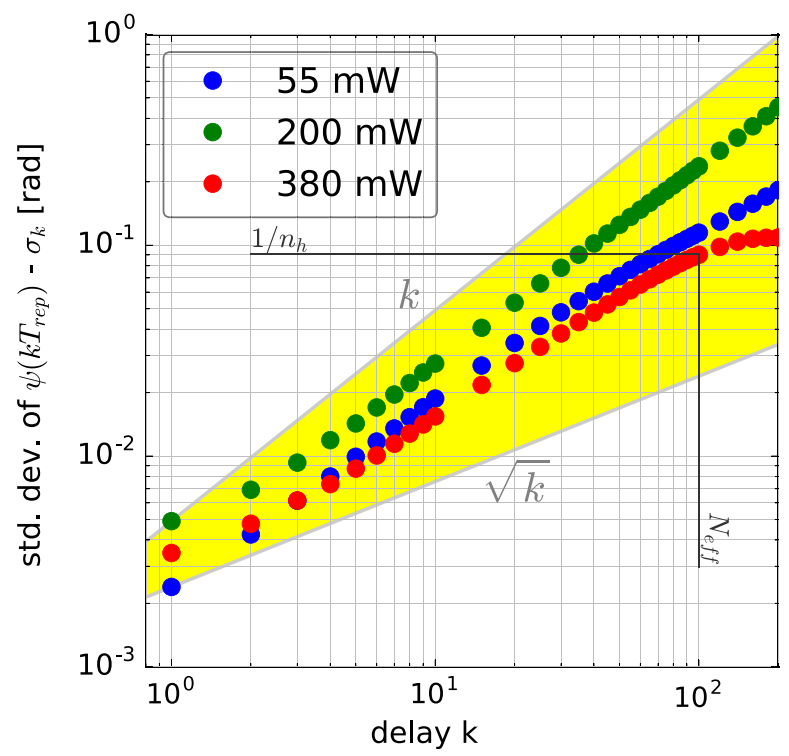

FIG. 3. Standard deviation $\sigma_{k}$ of the phase differences between two pulses separated by $k$ laser round trips as a function of $k$, for the three measurements of Fig. 2. The maximum number of coherent pulses $N_{\text {eff }}$ expected for an XUV comb at the harmonic order $n_{h}=11$ (for the $380 \mathrm{~mW}$ setting) is also indicated. cumulative diffusion would become significant for the fundamental radiation over $N_{\text {eff }} \approx 10^{3}-10^{4}$ subsequent pulses, as expected for a free-running OFC. By controlling this phase diffusion with a feedback loop, $N_{\text {eff }}$ could be greatly increased, as demonstrated in Ref. [14].

However, the situation is much more delicate when trying to generate a comb in the XUV via HHG, either directly or through accumulation in an enhancement cavity [31,32]. Our experimental Fig. 3 can be used to give a direct estimation of $N_{\text {eff }}$ in the XUV. In fact, since the coherence threshold for the $n_{h}$ harmonic is simply scaled down by the harmonic order, for $n_{h}=11$ one finds $N_{\text {eff }}=100$ at the maximum amplification setting. Since feedback loop times below $1 \mu \mathrm{s}$ are beyond current technical possibilities $[15,33]$, the driving IR OFC essentially behaves as a free-running (unreferenced) mode-locked laser in the context of HHG-based XUV spectroscopy and metrology. Therefore, the spectral width of an XUV comb line would be limited to about $\Delta \nu=\nu_{\text {rep }} / N_{\text {eff }} \approx 2.5 \mathrm{MHz}$, not far from the value estimated in [12]. Generating XUV tooth linewidths below this limit would require currently unavailable feedback loop times $T_{\text {loop }}<10^{-6} \mathrm{~s}$.

In conclusion, the pulse-to-pulse phase jitter of a commercial metrological $\mathrm{Er}^{+}$-doped fiber-based laser comb has been measured in different operating conditions, and found to range between 2 and $5 \mathrm{mrad}$. This very good phase stability confirms the quality of such a laser oscillator and its suitability as an OFC for high-accuracy frequency metrology with the available stabilization systems. More importantly, the measured pulse-to-pulse phase stability figure and its evolution over successive pulses is of crucial importance in the context of XUV comb-based spectroscopy with high-order harmonics, where it sets the minimum linewidth of XUV comb teeth. We believe that this direct measurement and analysis of the pulse-to-pulse phase jitter of an OFC will help clarify the current status of the field and identify the next significant hurdles towards the generation and application of XUV frequency combs.

This work was partially supported by LASERLABEUROPE (EU-H2020 654148). We gratefully acknowledge the skillful work of R. Ballerini and A. Hajeb, who designed and realized the vacuum chamber and the interferometer mechanical assembly. We also thank $\mathrm{M}$. Giuntini, M. De Pas, and A. Montori for their assistance in the setup of the electronics.

"Corresponding author. roberto.eramo@ino.it

'Present address: Attosecond Science Laboratory, King Saud University, Riyadh 11451, Saudi Arabia.

[1] S. A. Diddams, D. J. Jones, J. Ye, S. T. Cundiff, J. L. Hall, J. K. Ranka, R. S. Windeler, R. Holzwarth, T. Udem, and T. W. Hänsch, Phys. Rev. Lett. 84, 5102 (2000). 
[2] T. Udem, R. Holzwarth, and T. W. Hansch, Nature (London) 416, 233 (2002).

[3] J. L. Hall, Rev. Mod. Phys. 78, 1279 (2006).

[4] T. W. Hänsch, Rev. Mod. Phys. 78, 1297 (2006).

[5] P. Maddaloni, P. Cancio, and P. D. Natale, Meas. Sci. Technol. 20, 052001 (2009).

[6] S. A. Diddams, J. Opt. Soc. Am. B 27, B51 (2010).

[7] N. R. Newbury, Nat. Photonics 5, 186 (2011).

[8] C. Gohle, B. Stein, A. Schliesser, T. Udem, and T. W. Hänsch, Phys. Rev. Lett. 99, 263902 (2007).

[9] S. A. Diddams, L. Hollberg, and V. Mbele, Nature (London) 445, 627 (2007).

[10] B. Bernhardt, A. Ozawa, P. Jacquet, M. Jacquey, Y. Kobayashi, T. Udem, R. Holzwarth, G. Guelachvili, T. W. Hansch, and N. Picque, Nat. Photonics 4, 55 (2010).

[11] A. Foltynowicz, T. Ban, P. Masłowski, F. Adler, and J. Ye, Phys. Rev. Lett. 107, 233002 (2011).

[12] A. Cingöz, D. C. Yost, T. K. Allison, A. Ruehl, M. E. Fermann, I. Hartl, and J. Ye, Nature (London) 482, 68 (2012).

[13] M. Siciliani de Cumis, R. Eramo, N. Coluccelli, M. Cassinerio, G. Galzerano, P. Laporta, P. De Natale, and P. Cancio Pastor, Phys. Rev. A 91, 012505 (2015).

[14] T. R. Schibli, I. Hartl, D. C. Yost, M. J. Martin, A. Marcinkevicius, M. E. Fermann, and J. Ye, Nat. Photonics 2, 355 (2008).

[15] C. Benko, A. Ruehl, M. J. Martin, K. S. E. Eikema, M. E. Fermann, I. Hartl, and J. Ye, Opt. Lett. 37, 2196 (2012).

[16] E. E. Eyler, D. E. Chieda, M. C. Stowe, M. J. Thorpe, T. R. Schibli, and J. Ye, Eur. Phys. J. D 48, 43 (2008).

[17] B. Seiferle, L. von der Wense, and P. G. Thirolf, Phys. Rev. Lett. 118, 042501 (2017).

[18] See Supplemental Material at http://link.aps.org/ supplemental/10.1103/PhysRevLett.118.143201, which includes Refs. [19,20], for a discussion about the measurement of HHG based XUV comb coherence reported in literature, other details on our experimental set-up, and useful remarks about PSD and its link to the measured phase behaviour.

[19] C. Corsi, I. Liontos, S. Cavalieri, M. Bellini, G. Venturi, and R. Eramo, Opt. Express 23, 4106 (2015).

[20] G. R. Cooper and C. D. McGillem, Probabilistic Methods of Signal and System Analysis, 3rd ed. (Oxford University Press, New York, 1999).

[21] C. Benko, T. K. Allison, A. Cingoz, L. Hua, F. Labaye, D. C. Yost, and J. Ye, Nat. Photonics 8, 530 (2014).

[22] In the pre-OFC era, a measurement of the phase jitter PSD, at low Fourier frequencies (few MHz's) can be found in Ref. [23].

[23] L. Xu, C. Spielmann, A. Poppe, T. Brabec, F. Krausz, and T. W. Hänsch, Opt. Lett. 21, 2008 (1996).

[24] A full description will be published elsewhere.

[25] The neat PSD is obtained as the difference between the PSD of the I case and the NI case, see Supplemental Material at http://link.aps.org/supplemental/10.1103/PhysRevLett.118 .143201.

[26] S. Witte, R. T. Zinkstok, W. Ubachs, W. Hogervorst, and K. S. E. Eikema, Science 307, 400 (2005).

[27] D. Z. Kandula, C. Gohle, T. J. Pinkert, W. Ubachs, and K. S. E. Eikema, Phys. Rev. Lett. 105, 063001 (2010).

[28] S. Cavalieri and R. Eramo, Phys. Rev. A 58, R4263 (1998).

[29] S. Cavalieri, R. Eramo, M. Materazzi, C. Corsi, and M. Bellini, Phys. Rev. Lett. 89, 133002 (2002).

[30] R. Eramo, S. Cavalieri, C. Corsi, I. Liontos, and M. Bellini, Phys. Rev. Lett. 106, 213003 (2011).

[31] C. Gohle, T. Udem, M. Herrmann, J. Rauschenberger, R. Holzwarth, H. A. Schuessler, F. Krausz, and T. W. Hänsch, Nature (London) 436, 234 (2005).

[32] R. J. Jones, K. D. Moll, M. J. Thorpe, and J. Ye, Phys. Rev. Lett. 94, 193201 (2005).

[33] C.-C. Lee, C. Mohr, J. Bethge, S. Suzuki, M. E. Fermann, I. Hartl, and T. R. Schibli, Opt. Lett. 37, 3084 (2012). 